\title{
Direct enumeration of alloy configurations for electronic structural properties
}

\author{
Gus L. W. Hart \\ gus.hart@gmail.com \\ Peter A. Graf \\ Kwiseon Kim \\ Wesley B. Jones
}

Follow this and additional works at: https://scholarsarchive.byu.edu/facpub

Part of the Astrophysics and Astronomy Commons, and the Physics Commons

\section{Original Publication Citation}

Kwiseon Kim, Peter A. Graf, Wesley B. Jones and Gus L. W. Hart, "Direct enumeration of alloy configurations for electronic structural properties," Appl. Phys. Lett. 87, 243111 (8 Dec. 25). The original article may be found here: http://apl.aip.org/resource/1/applab/v87/i24/p243111_s1

\section{BYU ScholarsArchive Citation}

Hart, Gus L. W.; Graf, Peter A.; Kim, Kwiseon; and Jones, Wesley B., "Direct enumeration of alloy configurations for electronic structural properties" (2005). Faculty Publications. 347.

https://scholarsarchive.byu.edu/facpub/347 


\title{
Direct enumeration of alloy configurations for electronic structural properties
}

\author{
Peter A. Graf, Kwiseon Kim, ${ }^{\text {a) }}$ and Wesley B. Jones \\ National Renewable Energy Laboratory, Golden, Colorado 80401 \\ Gus L. W. Hart \\ Department of Physics, Northern Arizona University, Flagstaff, Arizona 86011-6010
}

(Received 23 June 2005; accepted 17 October 2005; published online 8 December 2005)

\begin{abstract}
We present and apply an approach to directly enumerate the band gaps and effective masses of all possible zinc blende-based alloy configurations whose unit cell contains up to a specified number of atoms. This method allows us to map the space of band gaps and effective masses versus alloy composition and atomic configuration. We demonstrate that a large number of band gaps and effective masses are available. We also discuss convergence of the method with respect to unit cell size and the combined optimization of band gap and effective mass for AlGaAs and GaInP semiconductor alloys. (C) 2005 American Institute of Physics. [DOI: 10.1063/1.2142091]
\end{abstract}

We address the atomistic design of materials with desired electronic properties. ${ }^{1,2}$ One obstacle in this search is the need to quickly scan the electronic properties of a large number of proposed materials. Here we present a straightforward but effective approach to this problem in which we enumerate all unique unit cells and bases derived from the faced centered cubic (fcc) lattice containing up to $N$ atoms and calculate the band gap and effective mass of each with a fast empirical pseudo-potential based electronic structure code. The method of direct enumeration has been used in the context of the ground state search of binary metal alloys. ${ }^{3}$ However, it has not been applied to electronic properties until now. In this letter we demonstrate the feasibility of a direct enumeration approach to the search for materials with desired electronic properties. The specific properties we focus on here are band gap and effective mass.

The specific space of structures we will examine is the space of mixed-cation pseudo-binary alloys, e.g., AlGaAs and GaInP. The possible materials represent different compositions and atomic configurations. For pseudo-binary alloys such as AlGaAs, attempts to treat the material design problem at the atomistic level founder on the fact that for a given number of atoms $N$, there are roughly $2^{N}$ structures to consider. So for systems of substantial size, only a small fraction of the available configurations are usually examined. The observation underlying our method is that the range of electronic structure properties of semiconductor alloys over all unit cells is approximately spanned by the range of properties over all unit cells of up to only $N$ atoms, where $N$ is rather small. The number of configurations for small $N$ is small enough that, combined with a fast electronic structure method, ${ }^{4}$ it becomes feasible to "search" by simply checking every possible configuration.

We work here exclusively with zinc blende alloys; we say "all possible configurations" with the understanding that we mean all possible zinc blende-based alloys. Making no assumptions about the configuration of the cations in a certain cell or about the cell shape, we consider all possible unit cells containing no more than $N$ cations, for $N \leqslant 8$. We are faced with a difficult problem in combinatorics-find all

\footnotetext{
${ }^{\text {a) }}$ Author to whom correspondence should be addressed; electronic mail:
} kwiseon_kim@nrel.gov possible binary structures on a fcc lattice with a given size unit cell. Fortunately, this problem was addressed earlier by Ferreira, Wei, and Zunger, who give a robust algorithm for generating a list of all possible binary structures on a fcc or body centered cubic lattice. ${ }^{3}$

Although the 629 structures generated by the abovementioned algorithm have small basis sizes, the unit cell vectors vary in direction. For example, many of the structures are [hkl] superlattices with none of $h, k, \ell$ equal to zero. The smallest fcc supercell containing all these structures commensurately is 4096 atom, $8 \times 8 \times 8 \mathrm{fcc}$ cell. This large cell, containing roughly $2^{2048}$ structures, is the size cell we would need to search to find all the cells of our enumeration in a search within a fixed fcc supercell. Thus, provided the structures we enumerate span the space of structures of interest, an issue we address below, our direct enumeration approach is vastly more efficient.

We have scanned the band gap as a function of alloy composition for $\mathrm{Al}_{x} \mathrm{Ga}_{1-x} \mathrm{As}$ and $\mathrm{Ga}_{x} \mathrm{In}_{1-x} \mathrm{P}$. The results are summarized in Figs. 1 and 2. The atomic configurations generated by our enumeration consist of a unit cell, a basis, and a list of which of the two cation species (e.g., Al and Ga) occupy which of the sites in the basis. For band gap, the unit cell is expanded by two in all directions in order to sample some of the zone edges, e.g., $X$ and $L$, with a single band structure calculation at $\Gamma^{2}$.

The atoms in the cell are relaxed according to a valence force field model, then atomic potentials are generated by the method of empirical pseudo potentials (EPM). The AlGaAs and GaInP EPM parameters used here are described in Refs. 5 and 6, respectively. The Schrödinger equation is solved by the folded spectrum method, which gives us the eigenvalues near the band edges. ${ }^{4}$ The fidelity of the empirical pseudo potentials used here is well established in Ref.s. 5-15.

Our first observation is that there is a wide spread of band gaps possible for any given composition $x$. Thus the picture of a bowing curve describing the band gap as a quadratic perturbation of a linear interpolation based on composition is incomplete. In fact, the space of possible band gaps forms a region. This dependence of band gaps on atomic ordering is partially known from experiment ${ }^{16}$ and previous theoretical work ${ }^{17}$ with more limited ranges of configura- 


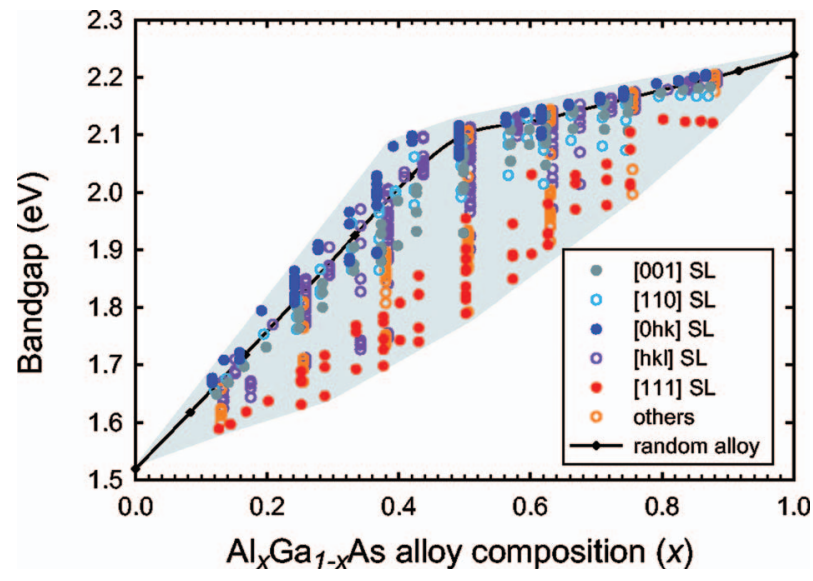

FIG. 1. (Color) Band gap vs composition for $\mathrm{Al}_{x} \mathrm{Ga}_{1-x} \mathrm{As}$ alloys. Every symbol (except for the random alloys) represents a unique unit cell with eight or less cation atoms. The random alloys are randomly created 12 $\times 12 \times 12$ (13824 atom) supercells. The different categories of superlattice are represented by different colors and symbols. We see clear trends; minimum band gap configurations are [111] superlattices, while maximum band gap configurations are [0hk] for $0<h \leqslant k$ and $k \geqslant 2$ or [ $h k \ell]$ for $0<h \leqslant k$ $\leqslant \ell$ and $k \geqslant 2$ superlattices. Note that the symbols for different SLs are slightly displaced in $x$ direction to enable visual distinction. The abscissa of each symbol should be read from the nearest red symbol.

tions, but our calculations confirm and extend these results to a wider range of configurations.

We find that a directional decomposition of the structures is most relevant. Depending on the periodicity in the multiples of zinc blende cell, different electronic states in the Brillouin zone interact with zone center states. ${ }^{18}$ In particular, the useful decomposition is found to be in terms of onedimensional (1D) superlattices (SLs). First, a division is made between [001], [111], [110] and more general directions. Structures that, although ordered, are not 1D superlattices are denoted "others." This decomposition is used throughout this letter.

Obvious trends emerge when the results are examined this way. Generally, $[0 h k]$ and $[h k \ell]$ SLs have higher band gaps than the rest. ${ }^{17}$ All the maximum band gap configurations for both AlGaAs and GaInP are of this form. Also, [111] SL's have lower band gaps than [001] SLs due to $L$ folding that repels the $\Gamma$ conduction band minimum to lower energies more than the $X$ folding. ${ }^{18}$ All the minimum band

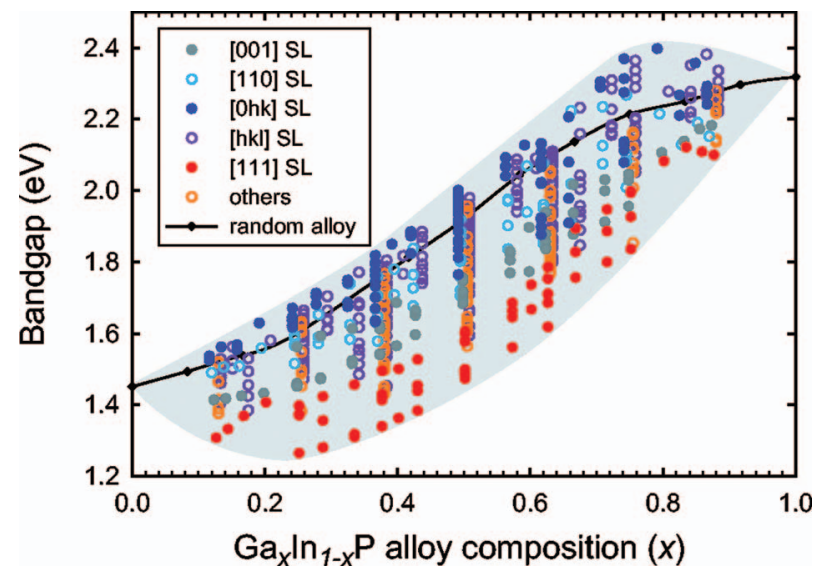

FIG. 2. (Color) Band gaps vs composition for "free-floating" $\operatorname{In}_{x} \mathrm{Ga}_{1-x} \mathrm{P}$ alloys. As in Fig. 1, every symbol represents a unique structure. Similar trends are evident. Again the minima are [111] superlattices. There is an indirect-direct transition near $x \sim 0.7$. which can be seen from the kink in the random alloy band gap line. random alloy band gap line.

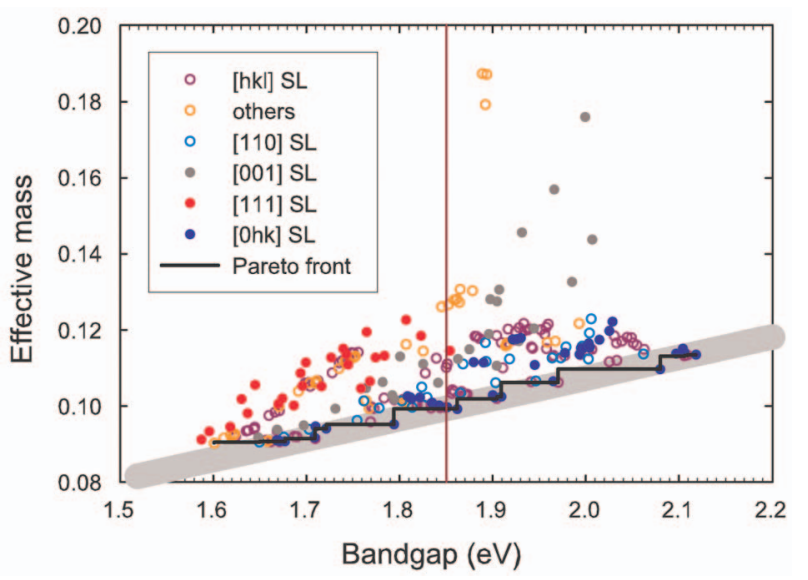

FIG. 3. (Color) Calculated electronic effective mass vs band gap for AlGaAs alloys. The Pareto front for the "minimum mass maximum gap" problem is indicated, as well as the $1.85 \mathrm{eV}$ line for the "minimum mass target gap" problem.

gap configurations except one is of this form. The exception, the $x=0.75$ minimum band gap AlGaAs structure, is not a superlattice. However, it is a structure with strong [111] ordering known as "Luzonite". 19 The spontaneous atomic ordering along [111] for GaInP was found to induce the reduction of band gap by $\sim 0.2-0.5 \mathrm{eV}^{16}$ relative to the random alloy consistent with our result in Fig. 2.

For AlGaAs, there is the well known direct to indirectat- $X$ transition at $x \sim 0.5$ in the random alloy curve. The bend due to this transition in the maximum gaps is visible. For the minima there is no visible transition because the conduction band minimum (CBM) of the minimum gap configuration is of the same character in all the [111] ordered structures.

For effective mass, we perform a series of calculations for different $k$ points near the $\Gamma$ point, compute the numerical second derivatives, and construct and diagonalize the effective mass tensor $m^{*}$. We take the geometric average and also record the anisotropy. We limit the electronic effective mass comparison to the $\mathrm{CBM}$ at $\Gamma$ in the composition range where for the random alloys the CBM is at $\Gamma$.

In Figs. 3 and 4 we plot the effective mass versus band gap for AlGaAs and GaInP, respectively. We find that the effective mass is approximated very well by a composition weighted average of the effective masses at the end points,

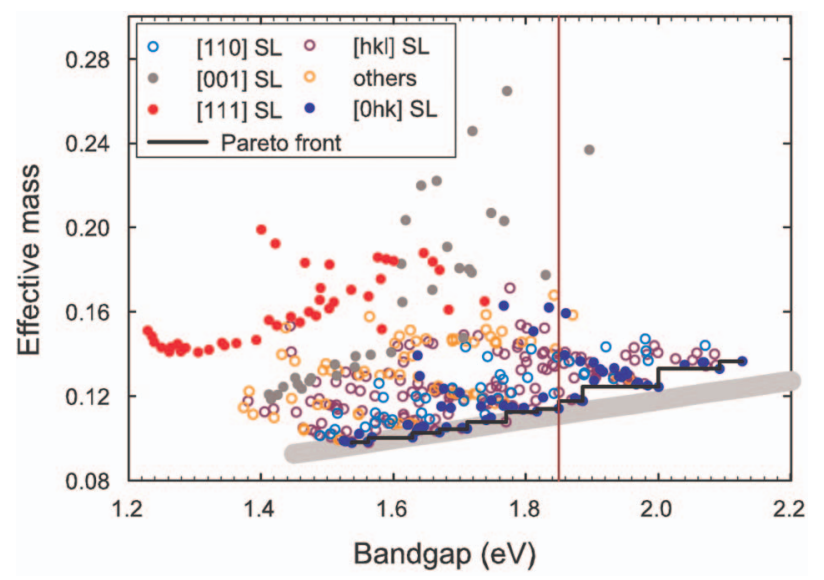

FIG. 4. (Color) Calculated electronic effective mass vs band gap for GaInP alloys. The Pareto front for the minimum mass maximum gap problem is indicated, as well as the $1.85 \mathrm{eV}$ line for the minimum mass target gap 
e.g., 0.16 for $\mathrm{AlAs}$ and 0.082 for GaAs calculated from the EPM parameters, ${ }^{5}$ except for the outliers mentioned below. Despite this near linear relationship versus composition, the effective mass versus band gap has a large spread above the linear average between the direct (i.e., at $\Gamma$ ) end point band gaps of 3.03 and $1.52 \mathrm{eV}$ for AlAs and GaAs, respectively. This average is shown as a thick gray line. The effective masses lie above the line due to the large range of reduced band gap versus a linear average as seen in Figs. 1 and 2 .

However, even in these compositions, certain configurations allow the folding of $X$ or $L$ to $\Gamma$, and the interaction of different zone edge states with the CBM at $\Gamma$ alters the effective masses. This explains the outliers with even larger masses than the end points. These represent structures with anisotropic masses having $X$-like effective mass properties and some larger isotropic masses. This behavior is observed in both AlGaAs and GaInP. The outlying values in AlGas typically occur for [001] superlattices and other, nonsuperlattice ordered structures. For GaInP they occur for [001] and [111] superlattices. This feature may be used in special applications such as in Ref. 20.

Suppose now that we wish to maximize the band gap at the same time as we minimize the effective mass. Attempts to optimize multiple properties generally involve a trade-off, since it is rare that both properties are optimized by the same solution. The set of points in a multicomponent search space which cannot be improved upon is known as the Pareto optimal set. ${ }^{21}$ The set of objective function values of the members of the Pareto optimal set is known as the Pareto Front. ${ }^{22}$ In Figs. 3 and 4 we have highlighted the Pareto front for the "minimum effective mass maximum band gap" question. We see there is no perfect solution, only a series of trade-offs.

As a further example, consider the question, "What is the configuration with band gap closest to $1.85 \mathrm{eV}$ and minimal effective mass?" This question arises in coated, surface modified photoelectrical chemical applications to hydrogen production, where the band gap must span the redox potential of water as well as satisfy other constraints. ${ }^{23}$ There is no unique answer to this question, since minimum effective mass and $1.85 \mathrm{eV}$ band gap do not occur in the same structure. In Figs. 3 and 4 we have indicated by a vertical line the structures with the desired band gap. Our method enables us to map the Pareto front for such problems in a straightforward way.

The relevance of this approach lies in the convergence of electronic properties as we increase the cell size. To investigate this issue we have studied the span of band gaps existing in the configurations for $N=4,8,12$, and 16. Figure 5 shows the results. The trend of ordering by superlattice is still evident. We see that already at $N=8$, the maximum values have converged to within a few meV. The minima for GaInP have also converged. The minima for AlGaAs do not converge. This is to be expected. The band gap of GaAs is the minimum over all compositions, so as the cell size grows, the minimum is achieved by a configuration with as much phase separation as the cell allows. On the other hand, the minimum band gap of GaInP does not occur at one of the end points, so the phase separation possible for larger cells does not result in lower band gaps.

To summarize, we have shown a method of rapidly scanning electronic properties of semiconductor alloys involving configurational degrees of freedom. We have shown an ex-

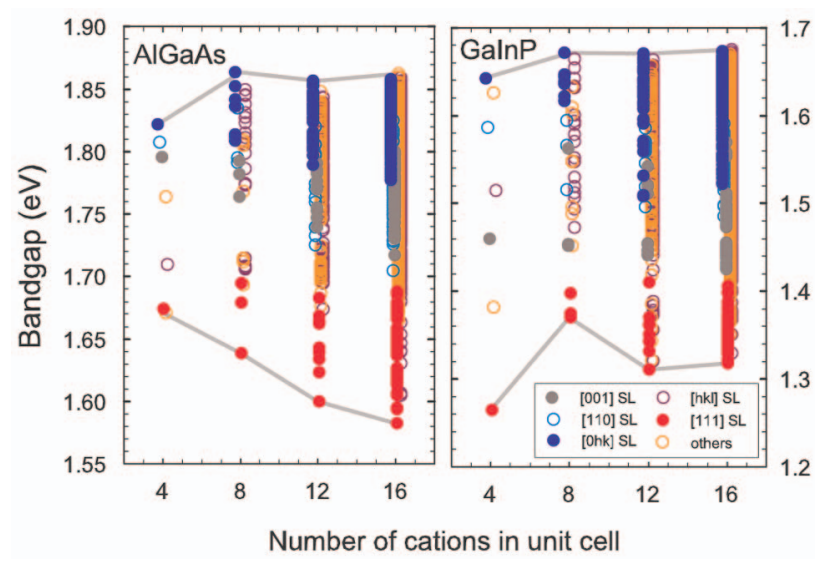

FIG. 5. (Color) Calculated band gaps vs number of cations in unit cell for $\mathrm{Al}_{0.25} \mathrm{Ga}_{0.75} \mathrm{As}$ alloys (left) and for $\mathrm{Ga}_{0.25} \mathrm{In}_{0.75} \mathrm{P}$ alloys (right). Same symbols as in Fig. 1. Solid gray lines mark the extrema. There are 7, 42, 391, and 2986 unique configurations in the unit cell consisting of $4,8,12$, and 16 cation atoms, respectively.

ample of combined optimization of two different electronic properties. We have presented evidence of the convergence of extrema with increasing unit cell size. Material properties design involving supercell calculations would benefit from the efficiency and effectiveness of this approach.

The authors thank L.-W. Wang for providing them with the PESCAN codes. Discussions with A. Zunger, G. Bester, S. Dudiy, V. Blum, Y. Zhang, and S.-H. Wei are greatly acknowledged. The authors acknowledge the computing resources at NERSC and at NREL-CSC. G.L.W.H. gratefully acknowleges support from the NSF under Grant No. DMR0244183. This work was supported by U.S. DOE-SC-ASCRMICS through Lab03-17 Theory Modeling in Nanoscience initiative under Contract No. DE-AC36-99GO10337.

${ }^{1}$ A. Franceschetti and A. Zunger, Nature (London) 402, 60 (1999).

${ }^{2}$ K. Kim, P. A. Graf, and W. B. Jones, J. Comput. Phys. 208, 735 (2005).

${ }^{3}$ L. G. Ferreira, S. H. Wei, and A. Zunger, Int. J. Supercomput. Appl. 5, 34 (1991).

${ }^{4}$ L.-W. Wang and A. Zunger, J. Chem. Phys. 100, 2394 (1994); A. Canning, L.-W. Wang, A. Williamson, and A. Zunger, J. Comput. Phys. 160, 29 (2000).

${ }^{5}$ K. A. Mäder and A. Zunger, Phys. Rev. B 50, 17393 (1994).

${ }^{6}$ T. Mattila, L.-W. Wang, and A. Zunger, Phys. Rev. B 59, 15270 (1999).

${ }^{7}$ K. A. Mäder and A. Zunger, Phys. Rev. B 51, 10462 (1995).

${ }^{8}$ K. A. Mäder, L.-W. Wang, and A. Zunger, Phys. Rev. Lett. 74, 2555 (1995).

${ }^{9}$ A. Franceschetti and A. Zunger, Phys. Rev. B 52, 14664 (1995).

${ }^{10}$ D. M. Wood and A. Zunger Phys. Rev. B 53, 7949 (1996).

${ }^{11}$ L. Wang and A. Zunger Phys. Rev. B 54, 11417 (1996).

${ }^{12}$ L.-W. Wang and A. Zunger, Phys. Rev. B 56, 12395 (1997).

${ }^{13}$ J. Kim, L.-W. Wang, and A. Zunger Phys. Rev. B 56, R15541 (1997)

${ }^{14}$ M. Sugisaki, H.-W. Ren, K. Nishi, and S. Sugou, Phys. Rev. B 61, 16040 (2000).

${ }^{15}$ T. Mattila, S.-H. Wei, and A. Zunger, Phys. Rev. Lett. 83, 2010 (1999).

${ }^{16}$ Y. Zhang, A. Mascarenhas, and L.-W. Wang, Phys. Rev. B 63, 201312(R) (2001); 64, 125207 (2001), Y. Zhang, A. Mascarenhas, and L.-W. Wang, Appl. Phys. Lett. 80, 3111 (2002).

${ }^{17}$ R. Magri and A. Zunger, Phys. Rev. B 44, 8672 (1991).

${ }^{18}$ S.-H. Wei and A. Zunger, Phys. Rev. B 39, 3279 (1989).

${ }^{19}$ S.-H. Wei and A. Zunger, J. Appl. Phys. 63, 5794 (1988).

${ }^{20}$ K. Vakili, Y. P. Shkolnikov, E. Tutuc, E. P. De Poortere, and M. Shayegan, Phys. Rev. Lett. 92, 186404 (2004); O. Gunawan, Y. P. Shokolnikov, E. P. De Poortere, E. Tutuc, and M. Shayegan, ibid. 93, 246603 (2004).

${ }^{21}$ C. M. Fonseca and P. J. Fleming, Evol. Comput. 3, 16 (1995).

${ }^{22}$ T. Bligaard, G. H. Jóhannesson, A. V. Ruban, H. L. Skriver, K. W. Jacobsen, and J. K. Nørkskov, Appl. Phys. Lett. 83, 4527 (2003).

${ }^{23}$ O. Khaselev and J. Turner, Science 280, 425 (1998). 